\title{
CONFORMAL HYPERSURFACES WITH THE SAME GAUSS MAP
}

\author{
MARCOS DAJCZER AND ENALDO VERGASTA
}

\begin{abstract}
In this paper we provide a complete classification of all hypersurfaces of Euclidean space which admit conformal deformations, other than the ones obtained through conformal diffeomorphisms of the ambient space, preserving the Gauss map.
\end{abstract}

Elie Cartan, in one of his earlier papers in differential geometry, classified all Euclidean hypersurfaces, of dimension at least five, which admit conformal deformations other than the trivial ones obtained through compositions with conformal diffeomorphisms of the ambient space. When all possible conformal deformations are trivial, a hypersurface is called conformally rigid. First, Cartan proved that a hypersurface is conformally rigid if at no point does there exist a principal curvature of multiplicity at least $n-2$ (see [Ca], [CD ${ }_{2}$, or [Da]). Using this, he concluded that a conformally deformable hypersurface is either conformally flat or a 2-parameter envelope of spheres or planes of some very special type. Moreover, the set of all conformal deformations is either a 1parameter family or there is just one other deformation.

In this paper we classify all Euclidean hypersurfaces which admit nontrivial conformal deformations preserving the Gauss map. In the isometric case, a complete classification for any codimension has been obtained by Dajczer and Gromoll [DG $\mathrm{DG}_{2}$, while the conformal case, but only for surfaces, is due to Vergasta $\left[\mathrm{Ve}_{2}\right]$.

Theorem. Let $f, g: M^{n} \rightarrow \mathbf{R}^{n+1}, n \geq 3$, be conformal immersions of an $n$ dimensional connected Riemannian manifold with the same Gauss map. Assume that on no open subset do they differ by a conformal diffeomorphism of $\mathbf{R}^{n+1}$. Then $f\left(M^{n}\right)$ is part of one of the following examples while $g\left(M^{n}\right)$ is of the same type:

(i) a minimal real Kaehler hypersurface,

(ii) a rotation hypersurface over a plane curve,

(iii) a rotation hypersurface over a minimal surface in $\mathbf{R}^{3}$.

Minimal real Kaehler hypersurfaces have been completely classified by Dajczer and Gromoll in [DG $\left.{ }_{2}\right]$. The assumption that $f$ and $g$ do not differ locally by a conformal deformation of the ambient space has been introduced in order to produce a global result. Without that assumption, our proof shows that the only other possibility is to have an open subset where, up to homothety

Received by the editors December 10, 1993.

1991 Mathematics Subject Classification. Primary 53C42. 
and rigid motion, either $f$ coincides with $g$ or $f$ is part of a cone and $g$ is obtained by an inversion with respect to tile vertex. When the cone is trivial, that is, part of an affine hyperplane, our statement reduces to the classical theorem of Liouville.

We conclude the paper discussing the deformations themselves. From now on all our classifications should be considered up to homothety and rigid motion. For hypersurfaces of type (i) or (iii), the set of nontrivial conformal deformations is a 1-parameter family. In fact, for hypersurfaces of type (i) we get the isometric associated family of minimal immersions defined in $\left[\mathrm{DG}_{2}\right]$. In case (iii), associated to the family of nonisometric deformations, what we obtain is a 1-parameter family of minimal surfaces such that the quotient of the distances to a fixed axis of any two of them is the conformal factor between the corresponding hypersurfaces. For each hypersurface of type (ii) there exists only one conformal deformation preserving the Gauss map.

Finally, only one hypersurface belongs simultaneously to two different classes, namely, the one in class (ii) obtained by rotating a catenary, which also belongs to class (iii). Its deformation as an element of class (ii) is the round sphere.

Some partial results related to the present work have been announced in $\left[\mathrm{Ve}_{1}\right]$. This work was completed while both authors were visiting the University of Granada. It is a pleasure to acknowledge the hospitality of the people at the Department of Geometry and Topology.

\section{THE PROOF OF THE THEOREM}

Let $f, g: M^{n} \rightarrow \mathbf{R}^{n+1}, n \geq 3$, be two conformal immersions with the same Gauss map. The conformal factor for the metric induced by $g$ with respect to the metric induced by $f$. is the smooth function $e^{\varphi}: M^{n} \rightarrow \mathbf{R}$ determined by

$$
\langle,\rangle_{g}=e^{2 \varphi}\langle,\rangle_{f}
$$

It has been shown in $\left[\mathrm{Ve}_{2}\right]$ that the map $T: T M \rightarrow T M$ given by

$$
T=e^{-\varphi} f_{*}^{-1} \circ P \circ g_{*},
$$

where $P$ stands for the parallel transport in $\mathbf{R}^{n+1}$, is an orthogonal tensor field. If we prefer to compare $f$ with respect to $g$, then the conformal factor is $e^{-\varphi}$ and the corresponding orthogonal tensor field is $T^{-1}$.

Recall that, by Cartan's result, when $n \geq 5$ and a nontrivial conformal deformation of $f$ exists, then at each point the second fundamental form has an umbilical or totally geodesic subspace of dimension at least $n-2$. To prove our Theorem, for each possible shape of the second fundamental form, we have to describe all admissible structures for the tensor field $T$.

We start with some basic results about the tensor field $T$. Replacing $f$ by $-f$ we see that any result for $T$ also holds for $-T$. In the sequel, with respect to the metric $\langle\rangle=,\langle,\rangle_{f}$, we denote by $\nabla$ the corresponding Levi-Civita connection and by $\nabla \varphi$ the gradient of $\varphi$.

Lemma 1. The tensor field $T$ satisfies the following differential equation:

$$
\left(\nabla_{X} T\right) Y=\langle Y, \nabla \varphi\rangle T X-\langle X, Y\rangle T \nabla \varphi, \quad \forall X, Y \in T M .
$$

Proof. This has been proved in [ $\left.\mathrm{Ve}_{2}\right]$.

We say that a vector subbundle $L$ of $T M$ is totally umbilical on $M^{n}$ if

$$
\left(\nabla_{X} Y\right)^{L^{\perp}}=\langle X, Y\rangle \eta
$$


for all $X, Y \in L$ and some fixed section $\eta$ of $L^{\perp}$. When $\nabla_{X} Y \in L$ for all $X, Y \in L$, we call $L$ totally geodesic.

Lemma 2. Let $L$ be a vector subbundle of $T M$ with $\operatorname{rank} L \geq 2$.

(i) If $\left.T\right|_{L}=I$, then $\nabla \varphi \in L^{\perp}$

(ii) If $L=\operatorname{ker}(T-I)$, then $L$ is integrable and totally umbilical while $L^{\perp}$ is totally geodesic.

Proof. Take $X, Y \in L$ to be orthogonal. We get from (1) that

$$
(I-T) \nabla_{X} Y=\langle Y, \nabla \varphi\rangle X,
$$

where the term on the left-hand side belongs to $L^{\perp}$. This proves (i).

For any $Y \in L=\operatorname{ker}(T-I)$ we have from (1) and (i) that

$$
(T-I) \nabla_{X} Y=\langle X, Y\rangle T \nabla \varphi
$$

for all $X \in T M$. It follows easily that

$$
\left(\nabla_{X} Y\right)^{L^{\perp}}=\langle X, Y\rangle \eta,
$$

where $\eta$ is the solution of $(T-I) \eta=T \nabla \varphi$.This implies (ii).

From now on, let us denote by $A$ (resp. $\bar{A}$ ) the second fundamental form (shape operator) for the immersion $f$ (resp. $g$ ) associated to a common unit normal local vector field.

Lemma 3. Suppose that the relative nullity subspaces $\Delta=\operatorname{ker} A$ form a subbundle of $T M$. Then

(i) $\Delta$ is $T$-invariant.

(ii) $\nabla \varphi \in \Delta$.

Proof. Part (i) is a consequence of the following relation proved in [ $\left[\mathrm{Ve}_{2}\right]$ :

$$
A \circ T=T^{-1} \circ A \text {. }
$$

For any unit vector field $X \in \Delta$ we have by (1)

$$
T \nabla \varphi=T \nabla_{X} X-\nabla_{X} T X+X(\varphi) T X .
$$

We conclude (ii) from (i) using that the relative nullity distribution $\Delta$ is totally geodesic.

Recall from [ $\left.\mathrm{Ve}_{2}\right]$ that if $M^{n}$ is simply connected, to any solution $\{\varphi, T\}$ of equation (1) satisfying (2) corresponds a unique conformal deformation preserving the Gauss map, given by the line integral

$$
g(x)=\int_{x_{0}}^{x} e^{\varphi} f_{*} \circ T .
$$

This has a consequence, which we will use several times, that any conformal deformation $g$ of $f$ preserving the Gauss map is completely determined by the pair $\{\varphi, T\}$.

From now on, we always assume that $f$ and $g$ do not differ by a conformal diffeomorphism of $\mathbf{R}^{n+1}$ on any open subset of $M^{n}$. This implies the following. 
Lemma 4. There is no open subset where $f$ is totally geodesic.

Proof. From $\left[\mathrm{Ve}_{2}\right]$ we have

$$
\bar{A}=e^{-\varphi} T^{-1} \circ A .
$$

Let $\mathscr{U}$ be an open subset of $M^{n}$ where $f$ is totally geodesic. From (3), the same holds for $g$. Hence, by Liouville's theorem, the restrictions of $f$ and $g$ to $\mathscr{U}$ must differ by a conformal diffeomorphism of the ambient space. But this possibility has been ruled out.

The case when the conformal factor is constant, that is, $f$ and $g$ are isometric up to homothety, has been considered in $\left[\mathrm{DG}_{2}\right]$. We state as a lemma the following consequence of the proof of Corollary 4.12 there.

Lemma 5. Suppose that $f$ and $g$ are isometric. Then $f$ and $g$ are associated minimal real Kaehler hypersurfaces. In particular, the tensor field $T$ has a single nonreal eigenvalue of multiplicity $n / 2$.

In the following four propositions our goal is to provide a complete geometric description whenever the second fundamental form of the hypersurface $f$ and the tensor field $T$ determined by $g$ are of some special forms. The proof of the Theorem will reduce to showing that, in fact, no other possibilities arise.

Proposition 6. Suppose that $T^{2}=I$. Then $f\left(M^{n}\right)$ is part of a rotation hypersurface over a plane curve.

Proof. From Lemma 5 we have that $\nabla \varphi \neq 0$ on an open dense subset. Moreover, by (i) of Lemma 2, the tangent bundle splits orthogonally and smoothly as

$$
T M=L^{n-1} \oplus L^{\perp},
$$

where, without loss of generality, we may assume $\left.T\right|_{L}=I$ and $\left.T\right|_{L^{\perp}}=-I$.

The leaves of $L$ are totally umbilical submanifolds of $M^{n}$ by Lemma 2. We claim that they are also totally umbilical in $\mathbf{R}^{n+1}$. Suppose, by contradiction, this not to be the case on an open connected subset $\mathscr{U} \subset M^{n}$. Given smooth unit vector fields $X \in L$ and $Y \in L^{\perp}$, we have from (1) and $\nabla \varphi \in L^{\perp}$ that

$$
Y(\varphi)=2\left\langle\nabla_{X} X, Y\right\rangle \text {. }
$$

Hence, for fixed $Y$ the smooth function $h=\left\langle\nabla_{X} X, Y\right\rangle$ is well defined. by

We show that the sectional curvature of the plane spanned by $X, Y$ is given

$$
K(X, Y)=Y(h)-h^{2} .
$$

Using Lemma 2, we have

$$
\nabla_{Y} Y=0, \quad \nabla_{X} Y=\left\langle\nabla_{X} Y, X\right\rangle X .
$$

Computing with respect to an orthonormal frame $X, Y, X_{1}, \ldots, X_{n-2}$, we get

$$
\begin{aligned}
K(X, Y)= & -\left\langle\nabla_{Y} \nabla_{X} Y, X\right\rangle-\left\langle\nabla_{\nabla_{X} Y} Y, X\right\rangle+\left\langle\nabla_{\nabla_{Y} X} Y, X\right\rangle \\
= & -Y\left(\left\langle\nabla_{X} Y, X\right\rangle\right)+\left\langle\nabla_{X} Y, \nabla_{Y} X\right\rangle-\left\langle\nabla_{X} Y, X\right\rangle^{2} \\
& +\sum_{i=1}^{n-2}\left\langle\nabla_{Y} X, X_{i}\right\rangle\left\langle\nabla_{X_{i}} Y, X\right\rangle \\
= & Y\left(\left\langle\nabla_{X} X, Y\right\rangle\right)-\left\langle\nabla_{X} X, Y\right\rangle^{2} .
\end{aligned}
$$


By equation (2), $L$ and $L^{\perp}$ are $A$-invariant. Thus, at any point $x \in$ $\mathscr{U}$ there exists an orthonormal basis $X_{1}, \ldots, X_{n-1}, Y$ of $T_{x} M$, with $L=$ $\operatorname{span}\left\{X_{1}, \ldots, X_{n-1}\right\}$, such that $A X_{j}=\lambda_{j} X_{j}$ and $A Y=\mu Y$. From the above, $\lambda_{i} \mu=\lambda_{j} \mu$ for $1 \leq i, j \leq n-1$. Then, from our assumption, $\mu=0$ along $\mathscr{U}$. Hence, by (6) the leaves of $L^{\perp}$ are geodesics of $\mathbf{R}^{n+1}$.

From (4) and (6), we have

$$
2 X(h)=X(Y(\varphi))=[X, Y](\varphi)=\nabla_{X} Y(\varphi)-\nabla_{Y} X(\varphi)=0,
$$

and from (5), we get

$$
Y(h)-h^{2}=0 .
$$

If $h$ vanishes at some point of $\mathscr{U}$, using equations (7) and (8) we easily conclude that $h$ must vanish on an open subset. Then, from (4), the same holds for $\nabla_{\varphi}$, which is not possible. Set $P=f+\frac{1}{h} Y$ on $\mathscr{U}$. We have using (6), (7) and $(8)$ that

$$
\widetilde{\nabla}_{X} P=X+\frac{1}{h} \widetilde{\nabla}_{X} Y=X+\frac{1}{h}\left\langle\nabla_{X} Y, X\right\rangle X=0,
$$

and

$$
\widetilde{\nabla}_{Y} P=Y-\frac{Y(h)}{h^{2}} Y=0,
$$

where $\widetilde{\nabla}$ denoves the standard flat connection on $\mathbf{R}^{n+1}$. This shows that $f(\mathscr{U})$ is locally part of a cone. Moreover, from (7) and (8), we have that the tensor field $T$ and the conformal factor are the correspondent to an inversion with center $P$. This has been excluded and proves the claim.

From the claim, $\left.A\right|_{L}=\lambda I$. We argue that the set $\left\{x \in M^{n}: \lambda(x) \neq 0\right\}$ is dense on $M^{n}$. We have seen, in Lemma 4, that $f$ is not totally geodesic on an open subset. Moreover, it can also not happen on an open subset that $\lambda=0$ and $\mu \neq 0$ because, in this case, we would have by Lemma 3 that $\nabla \varphi=0$ on this subset.

From the Codazzi equation and (6), we get

$$
X(\mu)=(\mu-\lambda)\left\langle\nabla_{Y} Y, X\right\rangle=0, \quad \forall X \in L .
$$

Moreover, from (4) and (6) we have

$$
\tilde{\nabla}_{X} Y=\nabla_{X} Y=-\frac{Y(\varphi)}{2} X
$$

Using the last two equations, it is easy to verify that the proof of Theorem 4.2 in $\left[\mathrm{CD}_{1}\right]$ still works and shows that $f\left(M^{n}\right)$ is part of a rotation hypersurface over a plane curve. In fact, a slightly more general argument than the one in $\left[C D_{1}\right]$ will be given at the end of the proof of Proposition 8.

Proposition 7. Suppose that the relative nullity spaces $\Delta=\operatorname{ker} A$ form a subbundle of TM of rank $n-2$. Then $f$ is a minimal real Kaehler hypersurface and $g$ an element of its associated family.

Proof. First we only assume that $\Delta$ is a proper vector subbundle of constant rank and prove that

$$
\left.T\right|_{\Delta^{\perp}} \neq \pm I
$$


Suppose, by contradiction, that $\left.T\right|_{\Delta^{\perp}}=I$. In particular, from Lemma $5, \nabla \varphi \neq$ 0 on an open dense subset of $M^{n}$. From now on we restrict ourselves to an open connected subset $\mathscr{U}$ of $M^{n}$ where $\nabla \varphi \neq 0$ and $L=\operatorname{ker}(T-I)$ is a vector subbundle of $T M$.

First suppose that $\operatorname{rank} \Delta \leq n-2$. Then $\operatorname{rank} L \geq 2$ since $\Delta^{\perp} \subset L$. Therefore, $\nabla \varphi \in L^{\perp}$ by Lemma 2 . Now suppose that $\operatorname{rank} \Delta=n-1$. In this case it may happen that $\operatorname{rank} L=1$. Thus, $L^{\perp}=\Delta$, and it is still true, from Lemma 3 , that $\nabla \varphi \in L^{\perp}$. From this and equation (1), we get

$$
T \nabla \varphi=(T-I) \nabla_{Y} Y, \quad \forall Y \in L,\|Y\|=1,
$$

and

$$
\nabla_{Z} Y \in L, \quad \forall Y \in L, \forall Z \perp Y .
$$

Equation (10) implies for any unit $Y \in L$ that $\left(\nabla_{Y} Y\right)^{L^{\perp}}$ never vanishes and does not depend on $Y$. Set

$$
V=L \oplus \operatorname{span}\left\{\left(\nabla_{Y} Y\right)^{L^{\perp}}\right\}
$$

We claim that both $V$ and $V^{\perp}$ are totally geodesic on $M^{n}$. Since $L$ is umbilical, to show that $V$ is totally geodesic it is sufficient to verify, by the use of (11), that $\nabla_{Z} \nabla_{Y} Y \in V$ for a fixed unit vector field $Y \in L$ and all $Z \in T M$. Let $\bar{Y} \in L$ be a unit vector field orthogonal to $Y$. From $R(\bar{Y}, Y) Y \in \Delta^{\perp} \subset L$ we have

$$
\nabla_{\bar{Y}} \nabla_{Y} Y-\nabla_{Y} \nabla_{\bar{Y}} Y-\nabla_{[\bar{Y}, Y]} Y \in L
$$

Since $\nabla_{\bar{Y}} Y \in L$ and $\left\langle Y, \nabla_{\bar{Y}} Y\right\rangle=0$, we get from (11) that $\nabla_{Y} \nabla_{\bar{Y}} Y \in L$. By similar arguments $\nabla_{[\bar{Y}, Y]} Y \in V$. Therefore, $\nabla_{\bar{Y}} \nabla_{Y} Y \in V$. Using that $R(X, Y) Y=0$ for $X \in \Delta$, an analogous argument when $X \perp Y$ yields $\nabla_{X} \nabla_{Y} Y \in V$.

To conclude the proof that $V$ is totally geodesic we have to show that $\nabla_{Y} \nabla_{Y} Y \in L$. From (11) and $\nabla \varphi \in L^{\perp}$,

$$
Y(X(\varphi))-\nabla_{Y} X(\varphi)=0, \quad \forall X \in L^{\perp} .
$$

On the other hand, by $(10)$

$$
Z(\varphi)=\left\langle\nabla_{Y} Y,(I-T) Z\right\rangle, \quad \forall Z \in T M .
$$

Consequently,

$$
Y\left(\left\langle\nabla_{Y} Y,(I-T) X\right\rangle\right)-\left\langle\nabla_{Y} Y,(I-T) \nabla_{Y} X\right\rangle=0 .
$$

After a short computation, we get

$$
\left\langle\nabla_{Y} \nabla_{Y} Y,(I-T) X\right\rangle=0, \quad \forall X \in L^{\perp} .
$$

Therefore, $\nabla_{Y} \nabla_{Y} Y \in L \subset V$ as we wished.

From (11) we have that $L^{\perp}$ is totally geodesic. To conclude that also $V^{\perp}$ is totally geodesic it is sufficient to show that $\left\langle\nabla_{X} \bar{X}, \nabla_{Y} Y\right\rangle=0$ for all $X, \bar{X} \in$ $V^{\perp}$. But this is clear from the fact we saw before that $\nabla_{X} \nabla_{Y} Y \in V$ for all $X \in T M$. This completes the proof of the claim.

Since $V^{\perp} \subset \Delta$, we obtain from the claim that $f(\mathscr{U})$ is locally contained in a Riemannian product $C^{k} \times \mathbf{R}^{n-k}$, where $k=\operatorname{rank} V$. Our next step is to show that $C^{k}$ is locally a cone. For a local unit vector field $Y \in L$, set

$$
\nabla_{Y} Y=\left(\nabla_{Y} Y\right)^{L}+\psi \eta, \quad \text { for } \eta=\frac{\left(\nabla_{Y} Y\right)^{L^{\perp}}}{\left\|\left(\nabla_{Y} Y\right)^{L^{\perp}}\right\|} .
$$


First we show that $\psi$ is constant along $L$. We have seen that

$$
\nabla_{Y} \nabla_{Y} Y=\nabla_{Y}\left(\nabla_{Y} Y\right)^{L}+Y(\psi) \eta+\psi \nabla_{Y} \eta \in L .
$$

By computing the $\eta$-component and using (11), we get that $Y(\psi)=0$. Since $\psi \eta$ is independent of $Y$, the statement follows.

Next we prove that $\eta(\psi)=\psi^{2}$. From (11) we have

$$
\left\langle\nabla_{\eta} \eta, Z\right\rangle=-\left\langle\nabla_{\eta} Z, \eta\right\rangle=0, \quad \forall Z \in L .
$$

From $\nabla_{\eta} \eta \in V$, we obtain the $\nabla_{\eta} \eta=0$. A straightforward computation of $\langle R(\eta, Y) Y, \eta\rangle=0$ using (11) yields the statement.

Set $P=f+\frac{1}{\psi} \eta$. Then

$$
\begin{gathered}
\tilde{\nabla}_{\eta} P=\eta+\eta(1 / \psi) \eta=0, \\
\widetilde{\nabla}_{Z} P=Z+\frac{1}{\psi} \widetilde{\nabla}_{Z} \eta=Z+\frac{1}{\psi}\left\langle\nabla_{Z} \eta, Z\right\rangle Z=.0, \quad \forall Z \in L,
\end{gathered}
$$

and we easily conclude that $C^{k}$ is locally a cone.

To compute the conformal factor for $g$ we consider a local parametrization

$$
f\left(s, y_{1}, \ldots, y_{k-1}, t_{1}, \ldots, t_{n-k}\right)=P+s \eta\left(y_{1}, \ldots, y_{k-1}\right)+\sum_{j=1}^{n-k} t_{j} E_{j},
$$

where the $E_{j}$ 's are constant in the ambient space and $\psi=-1 / s$. By (1), we have

$$
E_{j}(\varphi)=\frac{1}{s}\left\langle T E_{j}, \eta\right\rangle, \quad j=1, \ldots, n-k,
$$

and

$$
\eta(\varphi)=\frac{1}{s}(\langle T \eta, \eta\rangle-1) .
$$

From (1) and (13), we get

$$
s \nabla_{E_{j}} T \eta=(\langle T \eta, \eta\rangle-1) T E_{j} .
$$

Therefore,

$$
s(\langle T \eta, \eta\rangle)_{t_{j}}=(\langle T \eta, \eta\rangle-1)\left\langle T E_{j}, \eta\right\rangle .
$$

In particular, $\langle T \eta, \eta\rangle \neq 0$ on a dense subset because otherwise $\left\langle T E_{j}, \eta\right\rangle=0$, which is clearly not possible.

From (1), $\nabla_{\eta} T E_{j}=E_{j}(\varphi) T \eta$, which together with (12) yields

$$
s \nabla_{\eta} T E_{j}=\left\langle T E_{j}, \eta\right\rangle T \eta .
$$

We easily obtain that

$$
s\left(\left\langle T E_{j}, \eta\right\rangle^{2}\right)_{s}=2\left\langle T E_{j}, \eta\right\rangle^{2}\langle T \eta, \eta\rangle .
$$

By the orthogonality of $T$,

$$
\sum_{j=1}^{n-k}\left\langle T E_{j}, \eta\right\rangle^{2}=1-\langle T \eta, \eta\rangle^{2}
$$


Summing up equations (15) for $1 \leq j \leq n-k$ and using (16), we get

$$
\langle T \eta, \eta\rangle_{s}=\frac{1}{s}\left(\langle T \eta, \eta\rangle^{2}-1\right) .
$$

Therefore,

$$
\langle T \eta, \eta\rangle=\frac{\gamma-s^{2}}{\gamma+s^{2}}
$$

for some function $\gamma=\gamma\left(t_{1}, \ldots, t_{n-k}\right)$. We conclude from (13) and (17) that

$$
\eta(\varphi)=-\frac{2 s}{\gamma+s^{2}} .
$$

Equations (14) and (17) imply that

$$
\left\langle T E_{j}, \eta\right\rangle=-\frac{s \gamma_{t_{j}}}{\gamma+s^{2}} .
$$

Thus, by (12)

$$
E_{j}(\varphi)=-\frac{\gamma_{t_{j}}}{\gamma+s^{2}} .
$$

From (18) and (20), we have

$$
e^{\varphi}=\frac{a}{\gamma+s^{2}}
$$

for some constant $a \in \mathbf{R}$. In fact, composing $f$ with a homothety, if necessary, we may assume that $a=1$.

To obtain a contradiction we have to show that $f$ and $g$ agree locally by a conformal diffeomorphism of the ambient space. First observe that $g$ has exactly the same geometric description as $f$. More precisely, $f$ and $g$ are locally products of cones $f^{1}$ and $g^{1}$ of spherical immersions of a Riemannian manifold $N^{k}$, respectively, by an Euclidean factor $\mathbf{R}^{n-k}$. This is easily obtained by reasoning with $g$ instead of $f$ and using that $L=\operatorname{ker}\left(T^{-1}-I\right)$.

We claim that $\gamma(0)=0$. For the Levi-Civita connection $\bar{\nabla}$ of the metric induced by $g$, it is a standard fact that

$$
\bar{\nabla}_{Y} Y=\nabla_{Y} Y-\nabla \varphi \text {. }
$$

We have seen that the $L^{\perp}$-component of $\bar{\nabla}_{Y} Y$ must have the direction of a geodesic line. Taking local coordinates as before and using the expressions of $\psi$ and $\nabla \varphi$ in these coordinates along $t_{1}=\cdots=t_{n-k}=0$, we have

$$
\psi \eta-\nabla \varphi=\left(-\frac{1}{s}+\frac{2 s}{\gamma(0)+s^{2}}\right) \eta+\frac{1}{\gamma(0)+s^{2}} \nabla \gamma(0) .
$$

An easy computation shows that the vector fields $\psi \eta-\nabla \varphi,(\psi \eta-\nabla \varphi)_{s}$ are linearly dependent if and only if $\nabla \gamma(0)=0$. On the other hand, by (17) and (19), equation (16) becomes

$$
\|\nabla \gamma\|^{2}=4 \gamma,
$$

from which we conclude that $\gamma(0)=0$, as we wanted.

Using (17) we get that $T \eta=-\eta$ along $t_{1}=\cdots=t_{n-k}=0$. Since $\varphi$ and $T$ determine completely the deformation, we see up to homothety and isometric 
congruence that $g^{1}$ is the composition of $f^{1}$ with an inversion with respect to the vertex of the cone. On the other hand, from equation (1)

$$
\left(\left.\nabla_{Z} T\right|_{L^{\perp}}\right)^{L^{\perp}}=0, \quad \forall Z \in L .
$$

If $n-k \geq 3$, by Liouville's theorem, $T$ restricted to each fiber of $L^{\perp}$ must be, up to the conformal factor $e^{\varphi}=1 / s^{2}$, the differential of an inversion composed with an orthogonal transformation. By the above, the inversion must be taken with respect to the vertex of the cone and the orthogonal transformation acts on the Euclidean factor $\mathbf{R}^{n-k}$. Moreover, the orthogonal transformation is constant along $N^{k}$ because of (21). The same result holds for $n-k=2$. In this case, $\left(1 / s^{2}\right) T$ is the differential of either a holomorphic or antiholomorphic function defined on an open subset of a plane, and the remainder of the argument is straightforward. In both cases, we conclude that $g$ differs from $f$ by a composition of a rigid motion with this inversion. This concludes the proof of (9).

From now on we assume that $\Delta$ has rank $n-2$, and show that

$$
\operatorname{det}\left(\left.T\right|_{\Delta^{\perp}}\right)=1 \text {. }
$$

Let us suppose, by contradiction, that there exist smooth local vector fields $Y_{1}, Y_{2} \in \Delta^{\perp}$ such that $T Y_{1}=Y_{1}$ and $T Y_{2}=-Y_{2}$. From (1) and Lemma 3, we get

$$
\nabla_{Y_{1}} Y_{1}-T \nabla_{Y_{1}} Y_{1}=-T \nabla \varphi=-\nabla_{Y_{2}} Y_{2}-T \nabla_{Y_{2}} Y_{2}
$$

Hence,

$$
\left\langle\nabla_{Y_{1}} Y_{1}, Y_{2}\right\rangle=0=\left\langle\nabla_{Y_{2}} Y_{2}, Y_{1}\right\rangle
$$

We conclude that $\nabla_{Y_{i}} Y_{j} \in \Delta, 1 \leq i, j \leq 2$. On the other hand, from (2) we have that $A Y_{i}=\mu_{i} Y_{i}$, where $\mu_{1} \mu_{2} \neq 0$, everywhere. Then, the Codazzi equations imply

$$
\mu_{2}\left\langle\nabla_{Y_{1}} Y_{2}, X\right\rangle=\mu_{1}\left\langle\nabla_{Y_{2}} Y_{1}, X\right\rangle, \quad \forall X \in \Delta
$$

Thus $\nabla_{Y_{1}} Y_{2}$ and $\nabla_{Y_{2}} Y_{1}$ are always linearly dependent. But by (1)

$$
T \nabla_{Y_{1}} Y_{2}=-\nabla_{Y_{1}} Y_{2}, \quad T \nabla_{Y_{2}} Y_{1}=\nabla_{Y_{2}} Y_{1} \text {. }
$$

Hence, $\nabla_{Y_{1}} Y_{2}=\nabla_{Y_{2}} Y_{1}=0$. Therefore,

$$
\mu_{1} \mu_{2}=\left\langle R\left(Y_{1}, Y_{2}\right) Y_{2}, Y_{1}\right\rangle=-\left\langle\nabla_{Y_{1}} Y_{1}, \nabla_{Y_{2}} Y_{2}\right\rangle
$$

From (23),

$$
T\left(\nabla_{Y_{1}} Y_{1}-\nabla_{Y_{2}} Y_{2}\right)=\nabla_{Y_{1}} Y_{1}+\nabla_{Y_{2}} Y_{2}
$$

The orthogonality of $T$ implies that the last inner product in (24) vanishes. This is a contradiction and concludes the proof of (22).

All we have to show to obtain the proof of the proposition from Lemma 5 is that $\nabla \varphi=0$. From (22) we have that $\left.T\right|_{\Delta^{\perp}}$ is a rotation $R_{\theta}$ for some smooth function $\theta$. Hence,

$$
T Y_{1}=\cos \theta Y_{1}+\sin \theta Y_{2}, \quad T Y_{2}=-\sin \theta Y_{1}+\cos \theta Y_{2},
$$

where $Y_{1}, Y_{2} \in \Delta^{\perp}$ are smooth orthonormal vector fields such that

$$
A Y_{1}=\mu Y_{1}, \quad A Y_{2}=-\mu Y_{2} .
$$


From the Codazzi equations for any $X \in \Delta$,

$$
\begin{gathered}
\left\langle\nabla_{Y_{1}} Y_{1}, X\right\rangle=\frac{X(\mu)}{\mu}=\left\langle\nabla_{Y_{2}} Y_{2}, X\right\rangle, \\
\left\langle\nabla_{Y_{1}} Y_{2}, X\right\rangle+\left\langle\nabla_{Y_{2}} Y_{1}, X\right\rangle=0 .
\end{gathered}
$$

In particular, the $\Delta$-component of $\nabla_{Y} Y$ is independent of the unit vector field $Y \in \Delta^{\perp}$. Set

$$
\left(\nabla_{Y} Y\right)^{\Delta}=\phi X_{0}, \quad\left(\nabla_{Y_{1}} Y_{2}\right)^{\Delta}=-\left(\nabla_{Y_{2}} Y_{1}\right)^{\Delta}=\gamma X_{1},
$$

where $X_{0}, X_{1}$ are unit vector fields. In particular,

$$
\gamma T X_{1}=\left(T \nabla_{Y_{1}} Y_{2}\right)^{\Delta}=\left(\nabla_{Y_{1}} T Y_{2}\right)^{\Delta}=-\phi \sin \theta X_{0}+\gamma \cos \theta X_{1} .
$$

We claim that $\theta=$ constant,$\phi^{2}=\gamma^{2}$ and $\left\langle X_{0}, X_{1}\right\rangle=0$. From (1), we have

$$
\nabla_{X} T Y_{1}-T \nabla_{X} Y_{1}=0, \quad \forall X \in \Delta, \quad T \nabla \varphi=T \nabla_{Y_{i}} Y_{i}-\nabla_{Y_{i}} T Y_{i}, \quad i=1,2 .
$$

By the preceding equations we have that $\theta$ is constant and

$$
T \nabla \varphi=\phi T X_{0}-\phi \cos \theta X_{0}-\gamma \sin \theta X_{1} .
$$

By taking the inner product of (26) with $\gamma T X_{1}$ and using

$$
0=\left[Y_{1}, Y_{2}\right](\varphi)=\nabla_{Y_{1}} Y_{2}(\varphi)-\nabla_{Y_{2}} Y_{1}(\varphi)=2 \gamma X_{1}(\varphi),
$$

we get from (25)

$$
\left(\phi^{2}-\gamma^{2}\right) \cos \theta+2 \gamma \phi \sin \theta\left\langle X_{0}, X_{1}\right\rangle=0 .
$$

On the other hand, from (25)

$$
\gamma^{2}=\phi^{2} \sin ^{2} \theta+\gamma^{2} \cos ^{2} \theta-2 \phi \gamma \sin \theta \cos \theta\left\langle X_{0}, X_{1}\right\rangle,
$$

which together with (27) shows that $\phi^{2}=\gamma^{2}$. By (26), $\nabla \varphi=0$ at any point where $\phi=0$. Hence, we may restrict ourselves to the open subset where $\phi \neq 0$. We conclude that $\left\langle X_{0}, X_{1}\right\rangle=0$ from (27). This completes the proof of the claim.

We may assume $\phi=\gamma$ without loss of generality. From (26).

$$
T \nabla \varphi=\phi\left(T X_{0}-\beta\right),
$$

where $\beta=\cos \theta X_{0}+\sin \theta X_{1}$. Suppose that $T X_{0}-\beta \neq 0$ on a nonempty open subset. We claim that for all $Y \in \Delta^{\perp}$ it holds that

$$
\left(\nabla_{Y} \delta\right)^{\Delta}=0, \quad \text { where } \delta=\frac{T X_{0}-\beta}{\left\|T X_{0}-\beta\right\|} .
$$

Since $\Delta$ is totally geodesic, we have

$$
Y(X(\varphi))-\nabla_{Y} X(\varphi)=0, \quad \forall X \in \Delta, \forall Y \in \Delta^{\perp} .
$$

If in addition $X \perp \nabla \varphi$, we get using (28)

$$
\begin{aligned}
0 & =Y\langle X, \nabla \varphi\rangle=\left\langle\nabla_{Y} X, \nabla \varphi\right\rangle+\left\langle X, \nabla_{Y} \nabla \varphi\right\rangle \\
& =Y(X(\varphi))+\left\langle X, \nabla_{Y} \nabla_{\varphi}\right\rangle=\left\langle X, \nabla_{Y} \nabla \varphi\right\rangle .
\end{aligned}
$$

Thus,

$$
\left(\nabla_{Y} \nabla \varphi\right)^{\Delta} \in \operatorname{span}\{\nabla \varphi\}
$$


From (1)

$$
\left(\nabla_{Y} T \nabla \varphi\right)^{\Delta}=\left(T \nabla_{Y} \nabla \varphi\right)^{\Delta},
$$

and the claim follows easily from (29) and (30).

We recall from $\left[\mathrm{DG}_{1}\right]$ (see Lemma 2.2 ) that any minimal Euclidean hyperspace whose second fundamental form has constant rank 2 can be, at least locally, parametrized by the Gauss parametrization $\psi: \Lambda \rightarrow \mathbf{R}^{n+1}$ as

$$
\psi(x, w)=\gamma(x) h(x)+\nabla \gamma(x)+w .
$$

Here $\Lambda$ is the normal bundle of a minimal surface $h: N^{2} \rightarrow S_{1}^{n} \subset \mathbf{R}^{n+1}$ and $\gamma$ any smooth function on $N^{2}$ satisfying $\Delta \gamma+2 \gamma=0$. The fibers of $\Lambda$ can be identified by parallel translation in $\mathbf{R}^{n+1}$ to the leaves of the relative nullity foliation. In our case and by this identification, the vector fields $X_{0}, X_{1}$ and $\delta$ can be seen as normal vector fields to $h$. A similar argument as in the proof of Proposition 2.1 of $\left[\mathrm{DG}_{2}\right]$ shows that $X_{0}$ and $X_{1}$ span the first normal space $N_{h}^{1}$ of $h$ at each point. Recall that $N_{h}^{1}$ is just the normal subspace spanned by the image of the second fundamental form. So, in particular, $N_{h}^{1}$ is everywhere two dimensional. Moreover, under this new viewpoint, the claim we just proved says that $\delta$ is parallel in the normal connection of $h$. By definition, $\delta$ has a nonzero projection on $N_{h}^{1}$ at each point. From this and the Ricci equation, we easily conclude that $h$ must have flat normal bundle. But this is a contradiction since a minimal surface with flat normal bundle must satisfy $\operatorname{dim} N_{h}^{1}=1$.

Proposition 8. Suppose that $L=\operatorname{ker}\left(T^{2}-I\right)$ is a vector subbundle of rank $n-2$ such that the second fundamental form satisfies $\left.A\right|_{L}=\lambda I$ with $\lambda \neq 0$ everywhere. Then $f\left(M^{n}\right)$ is part of a rotational hypersurface over a minimal surface in $\mathbf{R}^{3}$. Proof. We claim that

$$
X(\varphi)=2\left\langle\nabla_{Y} Y, X\right\rangle, \quad \forall x \in L, \forall Y \in L^{\perp},\|Y\|=1 .
$$

From equation (1), we get

$$
X(\varphi)=\left\langle\nabla_{Y} Y, X\right\rangle-\left\langle\nabla_{Y} T Y, T X\right\rangle, \quad \forall X \in L, \forall Y \in L^{\perp},\|Y\|=1 .
$$

For any smooth local orthonormal basis $Y, Z \in L^{\perp}$ there exists a smooth function $\theta$ such that

$$
T Y=\cos \theta Y+\sin \theta Z, \quad T Z=-\sin \theta Y+\cos \theta Z .
$$

Hence, for all $X \in L$,

$$
\left\langle\nabla_{Y} T Y, X\right\rangle=\cos \theta\left\langle\nabla_{Y} Y, X\right\rangle+\sin \theta\left\langle\nabla_{Y} Z, X\right\rangle,
$$

and

$$
\left\langle\nabla_{Y} T Z, X\right\rangle=-\sin \theta\left\langle\nabla_{Y} Y, X\right\rangle+\cos \theta\left\langle\nabla_{Y} Z, X\right\rangle .
$$

Again from (1) we have

$$
\left\langle\nabla_{Y} T Z, T X\right\rangle-\left\langle\nabla_{Y} Z, X\right\rangle=0, \quad \forall X \in L,
$$

which together with (34) gives

$$
(\cos \theta \pm 1)\left\langle\nabla_{Y} Z, X\right\rangle=\sin \theta\left\langle\nabla_{Y} Y, X\right\rangle, \quad \text { if } T X=\mp X,
$$

and the claim follows from (32), (33) and (35). 
By (2) at any point there exist orthonormal vectors $Y_{1}, Y_{2} \in L^{\perp}$ such that

$$
A Y_{1}=\mu Y_{1}, \quad A Y_{2}=-\mu Y_{2} .
$$

On the open dense subset where the vector fields $Y_{1}, Y_{2}$ can be taken locally to be smooth, the Codazzi equations yield

$$
X(\mu)=(\mu-\lambda)\left\langle\nabla_{Y_{1}} Y_{1}, X\right\rangle=(\mu+\lambda)\left\langle\nabla_{Y_{2}} Y_{2}, X\right\rangle, \quad \forall X \in L .
$$

Clearly, from (31) and the above we have on any open subset where $\mu$ is smooth that

$$
\nabla \mu, \nabla \varphi \in L^{\perp} .
$$

We claim that $\left.T\right|_{L}= \pm I$, which is trivial for $n=3$. Suppose that, on some open subset, there exist smooth unit vector fields $X_{1}, X_{2} \in L$ such that $T X_{1}=X_{1}$ and $T X_{2}=-X_{2}$. From (1) and (36) we get

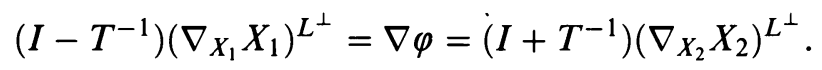

On the other hand, by the Codazzi equations we have, for any unit $X \in L$,

$$
Y_{1}(\lambda)=(\lambda-\mu)\left\langle\nabla_{X} X, Y_{1}\right\rangle, \quad Y_{2}(\lambda)=(\lambda+\mu)\left\langle\nabla_{X} X, Y_{2}\right\rangle .
$$

If on an open subset $\lambda \neq \pm \mu$, we obtain from (38) that $\left(\nabla_{X} X\right)^{L^{\perp}}$ is independent of $X$. From this and (37), we conclude that $\nabla \varphi=0$, which is a contradiction by Lemma 5 . If $\lambda= \pm \mu$ on an open subset, from (3) we have that both $e^{-\varphi} \lambda$ and $-e^{-\varphi} \lambda$ are eigenvalues of $\bar{A}$ with multiplicity at least 2 . Then the results in [Ry] imply that $g$ is totally geodesic on this subset. Thus the same happens with $f$, which contradicts Lemma 4 and proves the claim.

Without loss of generality we assume that $\left.T\right|_{L}=I$. By Lemma $2, L$ and $L^{\perp}$ are integrable distributions where $L^{\perp}$ is totally geodesic on $M^{n}$. In particular, since $L$ is $A$-invariant, we have that

$$
\widetilde{\nabla}_{Y} X \in L, \quad \forall X \in L, \forall Y \in L^{\perp} .
$$

Therefore, each leaf of $L^{\perp}$ is a surface in a 3-dimensional affine Euclidean space orthogonal to $L$.

If $n \geq 4$, from Lemma 2 and the hypothesis of the proposition, the leaves of $L$ are spheres. It remains to show that they are circles when $n=3$ which we will do later. For unit vector fields $\xi$ normal to $f$ and $X \in L$, set

$$
\sigma=\lambda \xi+\left(\nabla_{X} X\right)^{L^{\perp}} .
$$

From (37) we get that $\left(\nabla_{X} X\right)^{L^{\perp}}$ is independent of $X \in L$. We prove next that the locus of centers of these spheres given by

$$
\Gamma=f+\frac{1}{\|\sigma\|^{2}} \sigma
$$

is contained in a fixed plane. Until the conclusion of the proof let $X \in L$ be of unit length. A straightforward computation of

$$
\left\langle R\left(Y_{1}, X\right) X, Y_{1}\right\rangle=\lambda \mu=-\left\langle R\left(Y_{2}, X\right) X, Y_{2}\right\rangle
$$

yields

$$
Y_{1}\left(\left\langle\nabla_{X} X, Y_{1}\right\rangle\right)=\lambda \mu+\left\langle\nabla_{X} X, Y_{1}\right\rangle^{2}+\left\langle\nabla_{X} X, Y_{2}\right\rangle\left\langle\nabla_{Y_{1}} Y_{1}, Y_{2}\right\rangle,
$$


and

$$
Y_{2}\left(\left\langle\nabla_{X} X, Y_{2}\right\rangle\right)=-\lambda \mu+\left\langle\nabla_{X} X, Y_{2}\right\rangle^{2}+\left\langle\nabla_{X} X, Y_{1}\right\rangle\left\langle\nabla_{Y_{2}} Y_{2}, Y_{1}\right\rangle
$$

By a similar way, from $\left\langle R\left(Y_{i}, X\right) X, Y_{j}\right\rangle=0, i \neq j$, we get

$$
Y_{i}\left(\left\langle\nabla_{X} X, Y_{j}\right\rangle\right)=\left\langle\nabla_{X} X, Y_{i}\right\rangle\left(\left\langle\nabla_{X} X, Y_{j}\right\rangle-\left\langle\nabla_{Y_{i}} Y_{i}, Y_{j}\right\rangle\right), \quad i \neq j .
$$

From (9), we have that $\mu \neq 0$. So the Codazzi equations yield $\left\langle\nabla_{X} Y_{1}, Y_{2}\right\rangle=$ 0 , which, together with Lemma 2 , gives

$$
\nabla_{X} Y_{i}=\left\langle\nabla_{X} Y_{i}, X\right\rangle X, \quad i=1,2 .
$$

In particular, by (36) and (42), we have

$$
X\left(Y_{i}(\varphi)\right)=0=X\left(Y_{i}(\mu)\right), \quad i=1,2,
$$

because $X\left(Y_{i}(\varphi)\right)=\nabla_{X} Y_{i}(\varphi)-\nabla_{Y_{i}} X(\varphi)=0$, and similarly for $\mu$.

From (1) and (36),

$$
\left\{\begin{array}{l}
X(\theta)=0, \\
Y_{1}(\theta)=(\cos \theta-1)\left\langle\nabla_{X} X, Y_{2}\right\rangle-\sin \theta\left\langle\nabla_{X} X, Y_{1}\right\rangle, \\
Y_{2}(\theta)=(1-\cos \theta)\left\langle\nabla_{X} X, Y_{1}\right\rangle-\sin \theta\left\langle\nabla_{X} X, Y_{2}\right\rangle,
\end{array}\right.
$$

By (43), we have

$$
\left\{\begin{array}{l}
(1-\cos \theta) X\left(\left\langle\nabla_{X} X, Y_{1}\right\rangle\right)-\sin \theta X\left(\left\langle\nabla_{X} X, Y_{2}\right\rangle\right)=0 \\
(1-\cos \theta) X\left(\left\langle\nabla_{X} X, Y_{2}\right\rangle\right)+\sin \theta X\left(\left\langle\nabla_{X} X, Y_{1}\right\rangle\right)=0
\end{array}\right.
$$

Therefore,

$$
X\left(\left\langle\nabla_{X} X, Y_{i}\right\rangle\right)=0, \quad i=1,2 .
$$

From the Codazzi equations,

$$
Y_{i}(\mu)=2 \mu\left\langle\nabla_{Y_{j}} Y_{j}, Y_{i}\right\rangle, \quad i \neq j .
$$

Thus, using also (36) and (42), we get

$$
2 \mu X\left(\left\langle\nabla_{Y_{j}} Y_{j}, Y_{i}\right\rangle\right)=X\left(Y_{i}(\mu)\right)=\nabla_{X} Y_{i}(\mu)-\nabla_{Y_{i}} X(\mu)=0
$$

Now an easy computation using also (36), (39), (42), and (44) shows that

$$
X(\lambda)=0, \quad \forall X \in L \text {. }
$$

After a straightforward computation, we obtain from (38), (39), (40), (41), (42), (44) and (45) that

$$
\widetilde{\nabla}_{Y_{j}} \sigma=\left\langle\nabla_{X} X, Y_{j}\right\rangle \sigma, \quad \widetilde{\nabla}_{X} \sigma=-\|\sigma\|^{2} X
$$

Set

$$
\eta_{i}=\left\langle\nabla_{X} X, Y_{i}\right\rangle \xi-\lambda Y_{i}, \quad i=1,2 .
$$

From (42), (44) and (45) we get that $\widetilde{\nabla}_{X} \eta_{i}=0$ for any $X \in L$. On the other hand, an easy calculation using (38), (39), (40) and (41) shows that $\widetilde{\nabla}_{Y_{j}} \eta_{i} \in$ $\Omega=\operatorname{span}\left\{\eta_{1}, \eta_{2}\right\}, 1 \leq i, j \leq 2$. Thus $\Omega$ is a fixed plane orthogonal to $L$ and $\sigma$. From (46), we obtain

$$
X\left(\|\sigma\|^{2}\right)=0, \quad Y_{j}\left(\|\sigma\|^{2}\right)=2\left\langle\nabla_{X} X, Y_{j}\right\rangle\|\sigma\|^{2} .
$$


At this point we are in position to show that for $n=3$ the integral curves of $L$ are circles. This follows from $\widetilde{\nabla}_{X} X=\sigma$ and the first equation in (47). From (46) and (47)

$$
\tilde{\nabla}_{X} \Gamma=0, \quad \widetilde{\nabla}_{Y_{j}} \Gamma=Y_{j}-\frac{\left\langle\nabla_{X} X, Y_{j}\right\rangle}{\|\sigma\|^{2}} \sigma, \quad j=1,2 .
$$

Since the vectors $\widetilde{\nabla}_{Y_{j}} \Gamma$ are orthogonal to $L$ and $\sigma$, we conclude that the centers of the spheres lie in $\Omega$.

Proposition 9. Assume that $n=4$. If $\operatorname{ker}\left(T^{2}-I\right)=0$ everywhere, then $f$ is a minimal real Kaehler hypersurface and $g$ an element of its associated family.

Proof. The tangent bundle splits orthogonally and smoothly as $T M=L_{1} \oplus L_{2}$, where the restriction of $T$ to each summand is a rotation. By (2) there exist at each point orthonormal vectors $X_{1}, X_{2} \in L_{1}$ and $Y_{1}, Y_{2} \in L_{2}$ such that

$$
A X_{1}=\lambda X_{1}, \quad A X_{2}=-\lambda X_{2}, \quad A Y_{1}=\mu Y_{1}, \quad A Y_{2}=-\mu Y_{2} \text {. }
$$

Since $g$ is minimal from (3), both immersions are real analytic. Suppose that the conclusion of the proposition does not hold. By Lemma 5, there does not exist a nonempty open subset where $\nabla \varphi=0$. From Lemma 4 and Proposition 7 , the open subset $\left\{x \in M^{n}: \lambda(x) \mu(x) \neq 0\right\}$ is dense. On this subset we can take the vector fields and functions in (48) to be smooth. Set

$$
\begin{array}{ll}
T X_{1}=\cos \alpha X_{1}+\sin \alpha X_{2}, & T X_{2}=-\sin \alpha X_{1}+\cos \alpha X_{2}, \\
T Y_{1}=\cos \theta Y_{1}+\sin \theta Y_{2}, & T Y_{2}=-\sin \theta Y_{1}+\cos \theta Y_{2},
\end{array}
$$

where $\sin \alpha \sin \theta \neq 0$ everywhere. From (1) we obtain

$$
\left\{\begin{aligned}
&\left.\begin{array}{r}
(\cos \alpha \cos \theta-1)\left(\Gamma_{11}^{1}\right. \\
-
\end{array} \quad+\Gamma_{22}^{1}\right)+\cos \alpha \sin \theta\left(\Gamma_{11}^{2}-\Gamma_{22}^{2}\right)+\sin \alpha \cos \theta\left(\Gamma_{12}^{1}+\Gamma_{21}^{1}\right)+\sin \alpha \sin \theta\left(\Gamma_{12}^{2}+\Gamma_{21}^{2}\right)=0 \\
&(\cos \alpha \cos \theta-1)\left(\Gamma_{11}^{2}-\Gamma_{22}^{2}\right)-\cos \alpha \sin \theta\left(\Gamma_{11}^{1}-\Gamma_{22}^{1}\right) \\
& \quad+\sin \alpha \cos \theta\left(\Gamma_{12}^{2}+\Gamma_{21}^{2}\right)-\sin \alpha \sin \theta\left(\Gamma_{12}^{1}+\Gamma_{21}^{1}\right)=0 \\
&(\cos \alpha \cos \theta-1) \Gamma_{12}^{1}+\cos \alpha \sin \theta \Gamma_{12}^{2}-\sin \alpha \cos \theta \Gamma_{11}^{1}-\sin \alpha \sin \theta \Gamma_{11}^{2}=0 \\
&(\cos \alpha \cos \theta-1) \Gamma_{12}^{2}-\cos \alpha \sin \theta \Gamma_{12}^{1}-\sin \alpha \cos \theta \Gamma_{11}^{2}+\sin \alpha \sin \theta \Gamma_{11}^{1}=0 \\
&(\cos \alpha \cos \theta-1) \Gamma_{21}^{1}+\cos \alpha \sin \theta \Gamma_{21}^{2}+\sin \alpha \cos \theta \Gamma_{22}^{1}+\sin \alpha \sin \theta \Gamma_{22}^{2}=0 \\
&(\cos \alpha \cos \theta-1) \Gamma_{21}^{2}-\cos \alpha \sin \theta \Gamma_{21}^{1}+\sin \alpha \cos \theta \Gamma_{22}^{2}-\sin \alpha \sin \theta \Gamma_{22}^{1}=0
\end{aligned}\right.
$$

where $\Gamma_{i j}^{k}=\left\langle\nabla_{X_{i}} X_{j}, Y_{k}\right\rangle$ for $1 \leq i, j, k \leq 2$.

From (49), we get

$$
(\cos \alpha-\cos \theta)\left(\Gamma_{12}^{j}+\Gamma_{21}^{j}\right)=0, \quad j=1,2 .
$$

Now, again from (1)

$$
X(\theta)=0, \quad \forall X \in L_{1}, \quad Y(\alpha)=0, \quad \forall Y \in L_{2},
$$

and

$$
X_{1}(\varphi)=X_{2}(\alpha), \quad X_{2}(\varphi)=-X_{1}(\alpha), \quad Y_{1}(\varphi)=Y_{2}(\theta), \quad Y_{2}(\varphi)=-Y_{1}(\theta) .
$$

This shows that $\nabla \varphi=0$ on any open subset where $\cos \alpha=\cos \theta$. Thus, by (50)

$$
\Gamma_{12}^{j}+\Gamma_{21}^{j}=0, \quad j=1,2 .
$$


On the other hand, by the Codazzi equations we have

$$
(\mu+\lambda) \Gamma_{i j}^{i}=(\mu-\lambda) \Gamma_{j i}^{i}, \quad i \neq j .
$$

From (49), (51) and (52) we obtain that $\Gamma_{j k}^{k}=0$ for $1 \leq i, j, k \leq 2$. Then, using (1) once more, we get that $Y(\varphi)=0$ for all $y \in L_{2}$. Analogously, we have $X(\varphi)=0$ for all $X \in L_{1}$. This is a contradiction.

Proof of the Theorem. First we restrict ourselves to the case $n \geq 5$. Moreover, we assume that the conformal nullity $\nu_{c}(x)$ of $f$ at $x \in M^{n}$, defined by

$$
\nu_{c}(x)=\max _{\lambda \in \mathbf{R}}\{\operatorname{dim} \operatorname{ker}(A(x)-\lambda I)\},
$$

is constant in $M^{n}$. Let us denote by $W$ the subbundle of $T M$ of rank $\nu_{c}$ where $A X=\lambda X$ for all $X \in W$. By Cartan's conformal rigidity theorem stated in the introduction we only have to consider three cases.

Case $\nu_{c}=n-2$. We assume further that either (a) $\lambda=0$ or (b) $\lambda \neq 0$, everywhere. In situation (a) we have from Proposition 7 that $f$ and $g$ are as in part (i) of the Theorem. We aim to show that under assumption (b) the hypersurface is as in part (iii) of the Theorem. By equation (2),

$$
A\left(T-T^{-1}\right) X=-\lambda\left(T-T^{-1}\right) X, \quad \forall X \in W .
$$

Thus, $\left(T-T^{-1}\right) X \in W^{\perp}$. Al we have to show is just that the linear transformation $S=T-T^{-1}: W \rightarrow W^{\perp}$ vanishes everywhere. In fact, if $S=0$ we have that $W \subset \operatorname{ker}\left(T^{2}-I\right)$. Moreover, if $W \neq \operatorname{ker}\left(T^{2}-I\right)$, we get that $T^{2}=I$ and conclude from Proposition 6 that $\nu_{c} \geq n-1$, which is not possible. So $W=\operatorname{ker}\left(T^{2}-I\right)$ and this case follows from Proposition 8 .

If the dimension of $\operatorname{Im} S$ is 2 on some open subset, we have from $W^{\perp}=$ $\operatorname{ker}(A+\lambda I)$ that $-\lambda$ is a principal curvature of multiplicity 2 . This is not possible by the results in [Ry]. It remains to consider the much more difficult case when $\operatorname{Im} S$ is a line bundle along an open subset $\mathscr{U} \subset M^{n}$. By (2) and (53) there exist local orthonormal vector fields $Y_{1} \in W, Y_{2} \in \operatorname{Im} S$ and $Z_{0} \in W^{\perp}$, such that the tangent bundle splits orthogonally and smoothly as

$$
T M=\operatorname{ker} S \oplus \operatorname{span}\left\{Y_{1}, Y_{2}, Z_{0}\right\},
$$

where $A Y_{1}=\lambda Y_{1}, A Y_{2}=-\lambda Y_{2}, A Z_{0}=\mu Z_{0}$ and $\mu \neq \lambda$ everywhere. Moreover, as above, $\mu \neq-\lambda$ on a dense subset of $\mathscr{U}$.

We want to verify that $T Z_{0}= \pm Z_{0}$. Since $\operatorname{ker} S$ is $T$-invariant, $(\operatorname{ker} S)^{\perp}$ is three-dimensional and $T^{2} \neq I$, there exists a unique, up to sign, unit $X_{0} \in$ $(\operatorname{ker} S)^{\perp}$ such that $T X_{0}= \pm X_{0}$. From (2) we have $T A X_{0}= \pm A X_{0}$. Hence, $X_{0}$ and $A X_{0}$ are linearly dependent and we conclude that $Z_{0}= \pm X_{0}$, as we wished.

Next, we show that

$$
\left\{x \in M^{n}: \mu(x) \neq 0\right\}=\text { dense. }
$$

Suppose otherwise. From the above, $V=\operatorname{ker}\left(T^{2}-I\right)=\operatorname{ker} S \oplus \operatorname{span}\left\{Z_{0}\right\}$. Thus, it follows easily from (31) that

$$
\left\langle\nabla_{Y_{1}} Y_{2}, Z_{0}\right\rangle+\left\langle\nabla_{Y_{2}} Y_{1}, Z_{0}\right\rangle=0
$$

By Lemma 3 and our assumption, $\nabla \varphi \in \operatorname{span}\left\{Z_{0}\right\}$. Hence, (55) implies

$$
0=\left[Y_{1}, Y_{2}\right](\varphi)=2\left\langle\nabla_{Y_{1}} Y_{2}, Z_{0}\right\rangle Z_{0}(\varphi)
$$


From (31), (35) and (56) we get that $\nabla \varphi=0$. This is in contradiction with Lemma 5.

Without loss of generality, we assume that $T Z_{0}=-Z_{0}$. Next we prove that

$$
\operatorname{ker} S=\operatorname{ker}(T-I) \text {. }
$$

To see this all we have to do is to show that

$$
K\left(Z_{0}, Y\right)=K(X, Y), \quad \forall X \in \operatorname{ker}(T+I), \forall Y \in V^{\perp},
$$

and then use that $\mu \neq \lambda \neq 0$ to conclude the statement.

From the Codazzi equations, we get

$$
0=X(\lambda)=2 \lambda\left\langle\nabla_{Y_{2}} Y_{2}, X\right\rangle, \quad \forall X \in \operatorname{ker} S,
$$

and

$$
(\lambda-\mu)\left\langle\nabla_{Y_{1}} Y_{1}, Z_{0}\right\rangle=Z_{0}(\lambda)=(\lambda+\mu)\left\langle\nabla_{Y_{2}} Y_{2}, Z_{0}\right\rangle .
$$

We obtain from the above equation, (31) and (54) that, along $\mathscr{U}$,

$$
\nabla \varphi \in V^{\perp} \text {. }
$$

Let $Z_{0}, Z_{1}, \ldots, Z_{n-3} \in V$ be local orthonormal vector fields such that $T Z_{i}= \pm Z_{i}, 0 \leq i \leq n-3$. From (1), we have

$$
\nabla_{Z_{i}} T Z_{j}-T \nabla_{Z_{i}} Z_{j}=0, \quad i \neq j .
$$

Thus,

$$
\left(\nabla_{Z_{i}} Z_{j}\right)^{V^{\perp}}=0, \quad i \neq j .
$$

On the other hand, by (31), (35) and (59) we have that $V^{\perp}$ is totally geodesic on $M^{n}$. A straightforward computation using this fact and (60) yields

$$
K\left(Z_{i}, Y\right)=Y\left(\left\langle\nabla_{Z_{i}} Z_{i}, Y\right\rangle\right)-\left\langle\left(\nabla_{Z_{i}} Z_{i}\right)^{V^{\perp}}, \nabla_{Y} Y\right\rangle-\left\langle\nabla_{Z_{i}} Z_{i}, Y\right\rangle^{2} .
$$

Now, if $X \in \operatorname{ker}(T+I)$, we get from (1) and (59) that

$$
(I+T) \nabla_{Z_{0}} Z_{0}=T \nabla \varphi=(I+T) \nabla_{X} X .
$$

Thus,

which proves (58).

$$
\left(\nabla_{Z_{0}} Z_{0}\right)^{V^{\perp}}=\left(\nabla_{X} X\right)^{V^{\perp}}
$$

$\left\langle\nabla_{Z_{0}} Z_{0}, X\right\rangle=\left\langle\nabla_{Y} Z_{0}, X\right\rangle=\left\langle\nabla_{X^{\prime}} Z_{0}, X\right\rangle=0, \quad \forall Y \in V^{\perp}, \forall X \perp X^{\prime} \in \operatorname{ker} S$.

Equations (1) and (59) imply that

$$
\nabla_{X} Z_{0}=0, \quad \forall X \in \operatorname{ker} S .
$$

From (61), (62) and the Gauss equations, we obtain

$$
\lambda \mu=\left\langle\nabla_{X} \nabla_{Z_{0}} Z_{0}, X\right\rangle=-\left\langle\nabla_{Z_{0}} Z_{0}, \nabla_{X} X\right\rangle
$$

for any $X \in \operatorname{ker} S$. On the other hand, from (1) and (59), we have

$$
T\left(\nabla_{X} X-\nabla_{Z_{0}} Z_{0}\right)=\nabla_{X} X+\nabla_{Z_{0}} Z_{0}
$$

By the orthogonality of $T$ we get that $\left\langle\nabla_{Z_{0}} Z_{0}, \nabla_{X} X\right\rangle=0$. Therefore, from (63), we obtain that $\mu=0$. This is a contradiction and proves that $S=0$. 
Case $\nu_{c}=n-1$. From (9), $\lambda \neq 0$ on a dense subset of $M^{n}$. Hence, by (53), we have that $\left(T-T^{-1}\right) X \in W^{\perp}$ for any $X \in W$. As before, set $S=T-T^{-1}: W \rightarrow W^{\perp}$. In addition, let us assume that either (a) $S=0$ or (b) $S \neq 0$ everywhere. If (a) holds, from Proposition 6, the hypersurface is as in part (ii) of the Theorem. If (b) occurs, the hypersurface is of type (iii) by Proposition 8.

Case $\nu_{c}=n$. In this trivial situation $f$ is part of a sphere which can be seen as a rotational hypersurface of a circle and (53) implies that $T^{2}=I$.

We have shown that, on any connected component of some open dense subset, the image of $f$ is part of either a real Kaehler minimal hypersurface, a rotational hypersurface over a plane curve or a rotational hypersurface over a minimal surface in a 3-dimensional Euclidean space. By Lemma 5 and the proofs of the preceding propositions, along each component we have only one of the following possibilities:

(A) $T M=L_{1} \oplus \cdots \oplus L_{n / 2}$, where $\left.T\right|_{L_{i}}=R_{\theta}$, rank $L_{i}=2$, and $\theta \in \mathbf{R}$ is constant,

(B) $T M=L \oplus L^{\perp}$, where $\left.T\right|_{L}=I,\left.T\right|_{L^{\perp}}=-I$ and $\operatorname{rank} L=n-1$,

(C) $T M=L \oplus L^{\perp}$, where $L=\operatorname{ker}\left(T^{2}-I\right)=\operatorname{ker}(T-I)$ and $\operatorname{rank} L=n-2$.

Clearly, any two of the above types cannot be smoothly attached. It follows that the tensor field $T$ has the same structure everywhere. We conclude that $f\left(M^{n}\right)$ is globally as either (i), (ii) or (iii) in the Theorem.

Next, we consider the case $n=4$. Assume further that the subspaces $L=$ $\operatorname{ker}\left(T^{2}-I\right)$ form a subbundle of $T M$. We have to distinguish between three possibilities: (a) $L=T M$, (b) $L=0$ or (c) $\operatorname{rank} L=2$. In the first case, by Proposition 6, $f\left(M^{n}\right)$ is of type (ii) in the Theorem. If (b) occurs, $f\left(M^{n}\right)$ is of type (i) by Proposition 9.

Finally, we assume that (c) holds. We claim that $L$ is umbilical but not totally geodesic in the ambient space. Assuming the claim, it follows from Proposition 8 that $f\left(M^{n}\right)$ is of type (iii) in the Theorem. By Proposition 7, neither $L=\Delta$ nor $L^{\perp}=\Delta$. From (2), we can take smooth local orthonormal vector fields $X_{1}, X_{2} \in L$ and $Y_{1}, Y_{2} \in L^{\perp}$, such that $T X_{i}= \pm X_{i}, A X_{i}=\lambda_{i} X_{i}, A Y_{1}=\mu Y_{1}$ and $A Y_{2}=-\mu Y_{2}$.

We show that $\left.T\right|_{L}= \pm I$. Suppose otherwise and assume that $T X_{1}=X_{1}$, $T X_{2}=-X_{2}$. From the Codazzi equations, we get

$$
\left(\mu-\lambda_{i}\right)\left\langle\nabla_{Y_{1}} Y_{1}, X_{i}\right\rangle=X_{i}(\mu)=\left(\mu+\lambda_{i}\right)\left\langle\nabla_{Y_{2}} Y_{2}, X_{i}\right\rangle, \quad i=1,2 .
$$

By the above and (31), we have

$$
\lambda_{i}\left\langle\nabla_{Y_{j}} Y_{j}, X_{i}\right\rangle=0, \quad i, j=1,2 .
$$

Suppose initially that $\lambda_{1} \lambda_{2} \neq 0$. From (31) and (64) it foliows that $\nabla \varphi \in L^{\perp}$. Hence, by (1), we obtain

$$
\nabla_{X_{1}} X_{2}=\nabla_{X_{2}} X_{1}=0 .
$$

Now, the Gauss equations imply

$$
\lambda_{1} \lambda_{2}=-\left\langle\nabla_{X_{1}} X_{1}, \nabla_{X_{2}} X_{2}\right\rangle .
$$

Again from (1), we get

$$
(T-I)\left(\nabla_{X_{1}} X_{1}\right)=(T+I)\left(\nabla_{X_{2}} X_{2}\right) .
$$


Since $T$ is orthogonal, the inner product in (65) must vanish. This is a contradiction and shows that $\lambda_{1} \lambda_{2}=0$ everywhere.

From the beginning of the proof of the claim there is no open subset of $M^{n}$ where $\lambda_{1}=\lambda_{2}=0$. Therefore, by (9), there is an open dense subset where $\lambda_{1}=0, \lambda_{2} \neq 0$ and $\mu \neq 0$. As in the proof of (54) we obtain that $\nabla \varphi=0$, which is a contradiction. Hence, we may assume $\left.T\right|_{L}=-1$, and using that $\nabla \varphi \in L^{ \pm}$, we conclude from (58) that $L$ is umbilical in the ambient space. This proves the claim.

We have to argue that the different cases above cannot be smoothly attached. To see this, first notice that in case (a) we have $\operatorname{det}(T)=-1$ while in the other cases $\operatorname{det}(T)=1$. Also observe that in situations (b) and (c) the immersions are real analytic. Then, we may use that in case (b) the immersion is minimal while in situation (c) the mean curvature does not vanish.

It remains to consider the case $n=3$. If $T^{2}=I$ along $M^{n}$, then $f\left(M^{n}\right)$ is of type (ii) in the Theorem. Otherwise, we have an open subset where $L=$ $\operatorname{ker}\left(T^{2}-I\right)$ is a subbundle of rank 1. From Lemma 4 and (9) there is no open subset where $f$ is either totally geodesic or $L^{\perp}=\Delta$. By equation (2), we may take local orthonormal vector fields $X \in L$ and $Y_{1}, Y_{2} \in L^{\perp}$, such that $A X=\lambda X, A Y_{1}=\mu Y_{1}$ and $A Y_{2}=-\mu Y_{2}$, where $\mu \neq 0, T X=X$ and $\left.T\right|_{L^{\perp}}=R_{\theta}$. Now, the same argument used to prove (54) shows that $\lambda \neq 0$ on a dense subset of $M^{n}$. From Proposition 8, we have that $f$ is of type (iii) in the Theorem along each connected component of the subset $\left\{x \in M^{n}: \lambda(x) \neq 0\right\}$.

Again we have to verify that hypersurfaces of types (ii) and (iii) cannot have a common boundary. If that happens, the leaves of $L^{\perp}$ are spheres on one side and a minimal surface on the other. Therefore, both hypersurfaces can only be attached along a leaf. In this case, analyzing the behavior of the tensor $T$ it is not difficult to see that we must have $\mu=0$, which is not possible.

To finish the proof of the Theorem we have to argue that $g\left(M^{n}\right)$ is, in any case, of the same type as $f\left(M^{n}\right)$. But this follows easily from the fact that the tensor field $T^{-1}$ has the same structure as $T$ and the vector subbundle $L$ in Proposition 8 is also umbilical but not totally geodesic for $g$ as follows using equation (3).

We conclude this paper proving the statements in the introduction about the set of conformal deformations. If $f$ is a rotation hypersurface without umbilical points, we have seen in the proof of Proposition 6 that $T$ must satisfy $\left.T\right|_{L}=I$, where $L$ is the maximal umbilical subbundle of $T M$. Furthermore, (4) determines the function $\varphi$. Hence, at most one deformation $g$ is possible. Let $(\alpha, \beta)$ be any parametrization for the plane curve that generates $f$. Then the desired deformation is the hypersurface obtained by the rotation of $(1 / \alpha, \bar{\beta})$, where

$$
\bar{\beta}=-\int_{x_{0}}^{x}\left(\beta^{\prime} / \alpha^{2}\right) d \tau .
$$

Notice that this deformation is well defined even if $f$ has umbilical points. In particular, if we take $(\sin t, \cos t)$ as the generated curve for the unit sphere, the resulting deformation is the hypersurface obtained by rotation of the catenary $(1 / \sin t, \log \tan (t / 2))$.

Now let $f$ be a rotational hypersurface over a minimal surface in $\mathbf{R}^{3}$ with conformal nullity $n-2$ everywhere. As in the proof of equation (31), take unit 
vector fields $X \in L$ and $Y_{1}, Y_{2} \in L^{\perp}$. A straightforward computation shows that equations (39), (40) and (41) are satisfied. These equations are precisely the integrability conditions of the system

$$
\left\{\begin{array}{l}
X(\theta)=0, \\
Y_{1}(\theta)=(\cos \theta-1)\left\langle\nabla_{X} X, Y_{2}\right\rangle-\sin \theta\left\langle\nabla_{X} X, Y_{1}\right\rangle, \\
Y_{2}(\theta)=(1-\cos \theta)\left\langle\nabla_{X} X, Y_{1}\right\rangle-\sin \theta\left\langle\nabla_{X} X, Y_{2}\right\rangle,
\end{array}\right.
$$

and show that $\theta$ is a harmonic function defined on the minimal surface that generates $f$. We argue that the system

$$
\nabla \varphi \in L^{\perp}, \quad Y_{1}(\varphi)=Y_{2}(\theta), \quad Y_{2}(\varphi)=-Y_{1}(\theta)
$$

is also integrable. In fact, the function $\varphi$ is harmonic conjugate to $f$. For each solution of (66) we define $T$ by $\left.T\right|_{L}=I$ and $\left.T\right|_{L^{\perp}}=R_{\theta}$, and take the solution of (67). Using (66) and (67) one can verify that $\{\varphi, T\}$ satisfies equation (1), while condition (2) follows directly from the definition of $T$. Notice that distinct solutions of (66) do not differ by a constant. So, restricted to any simple connected subset of $M^{n}$, we have precisely a 1-parameter family of deformations. For the proofs of Proposition 8 and the Theorem, one can see that any deformation $g$ must agree with one of the elements of this family. Also from (66) and (67) we have that in the family of deformations no one is isometric.

Now let $(a(y, z) \Phi(x), b(y, z), c(y, z))$ be a parametrization of $f$, where $\Phi=\Phi(x)$ is any parametrization of the $(n-2)$-dimensional unit sphere and $(a, b, c)$ is a minimal surface in isothermal coordinates. A solution of (66) is a function $\theta=\theta(y, z)$ which satisfies

$$
\left\{\begin{array}{l}
\theta_{y}=\left(a_{y} / a\right) \sin \theta+\left(a_{z} / a\right)(1-\cos \theta), \\
\theta_{z}=\left(a_{z} / a\right) \sin \theta-\left(a_{y} / a\right)(1-\cos \theta) .
\end{array}\right.
$$

For each $\theta$, the corresponding deformation $g$ can be parameterized by

$$
(\alpha(y, z) \Phi(x), \beta(y, z), \gamma(y, z)),
$$

where $(\alpha, \beta, \gamma)$ is a minimal surface in isothermal coordinates which verifies

$$
\left\{\begin{array}{l}
\alpha=a e^{\varphi}, \\
\beta_{y}=e^{\varphi}\left(\cos \theta b_{y}+\sin \theta b_{z}\right), \\
\beta_{z}=e^{\varphi}\left(-\sin \theta b_{y}+\cos \theta b_{z}\right), \\
\gamma_{y}=e^{\varphi}\left(\cos \theta c_{y}+\sin \theta b_{z}\right), \\
\gamma_{z}=e^{\varphi}\left(-\sin \theta c_{y}+\cos \theta c_{z}\right) .
\end{array}\right.
$$

So we get a conformal transformation between isothermal minimal surfaces which preserves the Gauss map and whose conformal factor is given by the quotient of the respective distance functions to a fixed axis.

\section{REFERENCES}

[Ca] E. Cartan, La déformation des hipersurfaces dans lespace conforme réel à $n \geq 5$ dimensions, Bull. Soc. Math. France 45 (1917), 57-121.

[CD $]$ M. do Carmo and M. Dajczer, Rotational hypersurfaces in spaces of constant curvature, Trans. Amer. Math. Soc. 277 (1983), 685-709. 
$\left[\mathrm{CD}_{2}\right] \_$, Conformal rigidity, Amer. J. Math. 109 (1983), 963-985.

[Da] M. Dajczer et al., Submanifolds and isometric immersions, Math. Lecture Series 13, Publish or Perish, Houston, 1990.

$\left[\mathrm{DG}_{1}\right]$ M. Dajczer and D. Gromoll, Gauss parametrizations and rigidity aspects of submanifolds, J. Differential Geom. 22 (1985), 1-12.

$\left[\mathrm{DG}_{2}\right] \ldots$, Real Kaehler submanifolds and uniqueness of the Gauss map, J. Differential Geom. 22 (1985), 13-28.

[Ry] P. Ryan, Homogeneity and curvature conditions for hypersurfaces, Tohôku Math. J. 21 (1969), 363-388.

[Ve $\mathrm{V}_{1}$ ] E. Vergasta, Conformal immersions with the same Gauss map, An. Acad. Brasil. Cienc. 59 (1987), 145-147.

[ $\left.\mathrm{Ve}_{2}\right]-$ Conformal deformations preserving the Gauss map, Pacific J. Math. 156 (1992), 359-369.

IMPA, Estrada Dona Castorina, 110, 22460-320 Rio de Janeiro, Brazil

E-mail address: marcos@impa.br

Universidade Federal da Bahia, 40210 Bahia, Brazil

E-mail address: vergasta@brufba.bitnet 